\title{
In vivo Confocal Laser Scanning Microscopy and Micropuncture in Intact Rat
}

\author{
Yoshio Ohno Henrik Birn Erik I. Christensen \\ Department of Cell Biology, Institute of Anatomy, University of Aarhus, Aarhus, Denmark
}

\section{Key Words}

Confocal laser scanning microscopy · Kidney proximal tubules · Intravital microscopy · Protein reabsorption • Endocytosis

\begin{abstract}
Background: Intravital microscopy theoretically provides the optimal conditions for studying specific organ functions. However, the application of microscopy in intact organs in vivo has been limited so far due to technical difficulties. The purpose of this study was to establish a method of in vivo confocal laser scanning microscopy (CLSM) for the study of endocytosis in proximal tubules of intact kidney. Methods: The left kidney of rats placed on a modified microscope stage was exposed and stabilized in a thermostatically controlled cup. The stage was then attached to an upright confocal microscope. Surface proximal tubules were microinfused with fluorescent albumin or transferrin. Single or time-series images of microinfused proximal tubules were recorded in reflection and/or fluorescence mode. Results: The stability of the kidney and the resolution of images were sufficient to visualize intracellular vesicles. Albumin and transferrin were initially observed at the brush border, then later internalized by proximal tubules and accumulated in lysosomes over a time period of $15 \mathrm{~min}$. Furthermore, fusion of vesicles was observed in time-lapse images. Conclusion: These results show that in vivo CLSM in intact kidney may be an excellent method to evaluate proximal tubular endocytosis and ligand trafficking.
\end{abstract}

Copyright $\odot 2005$ S. Karger AG, Basel

\section{Introduction}

Confocal laser scanning microscopy (CLSM) has been widely applied in cell biology studies over the past decade. The advantage of CLSM relies on the detector pinhole and the photo multiplier tube (PMT). The detector pinhole allows three-dimensional reconstruction of intracellular structures. The PMT helps to collect weak emitted light, multiple fluorescent signals, and to reduce laser power and concentration of fluorescent probes.

The study of living cells by CLSM has provided original intracellular spatial and temporal information. Technical developments of fluorescent probes including greenfluorescent protein [1] and its variants [2], and increased scanning speed of microscope systems have added significantly to studies of intracellular dynamics in living cells. So far, spatial distribution of cellular components [3], membrane dynamics [4], fusion [5] and transport of vesicles [6] have been visualized with time-lapse recording. Furthermore, fluorescence-tagged single particles can now be detected in time-lapse studies [7].

While the majority of these studies have been performed in vitro on cells in culture, the in vivo study of specific organ structural-functional relationships at the cellular level generates several difficulties. First, the observable depth of microscopy is limited by heterogeneity in refractive indexes between cytosol, cell membrane and interstitium. Second, organ movements caused by microcirculation and respiration may create problems, especially at higher magnifications. Few organs have been visualized by this technique: brain [8], spleen [9], skin [10], cor-

Erik Ilsø Christensen

Department of Cell Biology, University of Aarhus

University Park, Building 234

DK-8000 Aarhus C (Denmark)

Tel. +45 8942 3057, Fax +458619 8664, E-Mail eic@ana.au.dk 

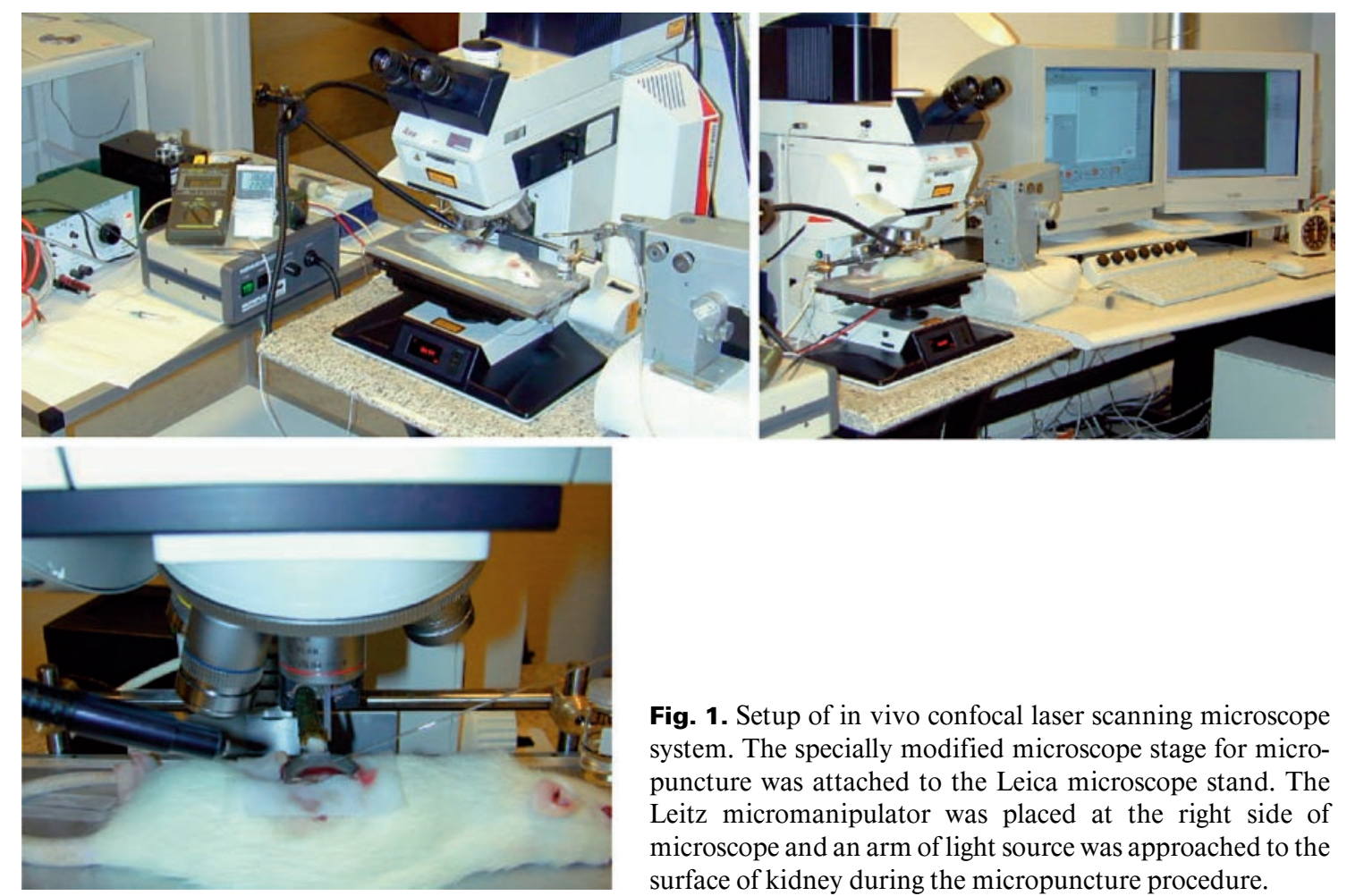

Fig. 1. Setup of in vivo confocal laser scanning microscope system. The specially modified microscope stage for micropuncture was attached to the Leica microscope stand. The Leitz micromanipulator was placed at the right side of microscope and an arm of light source was approached to the surface of kidney during the micropuncture procedure.

nea [11], lung [12], and kidney [13] and most of them for studies of morphological changes and hemodynamics in the intact organ. Recently, Dunn et al. [14] successfully studied the dynamics of receptor-mediated endocytosis in intact kidney by two-photon laser scanning microscopy (TPLSM) demonstrating individual endosomes containing fluorescence-labeled dextran and gentamicin.

The purpose of this study was to visualize the process of endocytosis in proximal tubular cells in rat intact kidney in nearly real time by CLSM. The kidney is easily accessible, undertakes rapid and efficient proximal tubule endocytosis, and established micropuncture techniques used intensively in our previous studies allow us to handle single nephrons without systemic effects $[15,16]$. Intact rat proximal tubules were microinfused with fluorescent rat serum albumin (RSA) or human transferrin (Tf) and analysed in situ by CLSM. By optimizing the technique we could visualize the endocytosis and lysosomal accumulation of microinjected RSA and Tf. Fusion of endocytic vesicles was demonstrated by time-lapse imaging. The observations suggest that in vivo confocal microscopy could become an important tool for the study of renal physiological and pathological processes.

\section{Materials and Methods}

\section{Setup}

All animal experiments were carried out in accordance with provisions for the animal care license provided by the Danish National Animal Experiments Inspectorate.

Male Wistar rats (200-300 g body weight) were starved overnight and anesthetized by intraperitoneal injection of $100 \mathrm{mg} / \mathrm{kg}$ of thiobutabarbital sodium (Inactin ${ }^{\circledR}$, Sigma Chemical Co., St. Louis, Mo., USA). Rats were placed on a modified microscope stage equipped with a thermostatically controlled system. The temperature of the stage was kept at $37^{\circ} \mathrm{C}$ during the experiment. Following tracheoto$\mathrm{my}$, the right jugular vein was cannulated for constant infusion of physiological saline at the rate of $3.8 \mathrm{ml} / \mathrm{h}$. The left kidney was exposed by a flank incision and placed in a thermostatically controlled cup. The kidney was stabilized in the cup by closing the empty space around the kidney with absorbent cotton. The cotton was stiffened with 3\% agar dissolved in saline and the surface of kidney was covered with paraffin oil. The stage was attached to a Leica DM RXE microscope stand (Leica Microsystems, Heidelberg, Germany). Setup of the experimental system is shown in figure 1 .

\section{Fluorescent Probes}

Rat serum albumin (RSA) (Sigma, essentially globulin free, A4538) and human holo-transferrin (Sigma, T4132) were labeled with Alexa Fluor 488 (Molecular Probe Europe BV, The Netherlands) according to manufacturer's instruction. In brief, RSA and Tf were dissolved at $2 \mathrm{mg} / \mathrm{ml}$ in phosphate-buffered saline, and $0.5 \mathrm{ml}$ of 
the solution was alkalized with $50 \mu 1$ of $1 M$ sodium bicarbonate solution. The mixture was reacted with Alexa Fluor 488 dye for $1 \mathrm{~h}$ at room temperature, while stirring. The labeled protein was purified by resin column chromatography. Labeling efficiency of RSA and Tf were 2.1:1 and 5.2:1, respectively. Absorption and fluorescence emission maximum were at 494 and $519 \mathrm{~nm}$, respectively.

\section{Micropuncture}

Micropuncture was conducted using a $4 \times$ objective lens and with the modified stage fixed to the confocal microscope stand. Alexa Fluor 488 labeled protein solutions were stained with Lissamine green (Sigma) and the final concentration of RSA and Tf were adjusted to $90 \mu \mathrm{g} / \mathrm{ml}\left(1.38 \times 10^{-6} M\right)$ and $60 \mu \mathrm{g} / \mathrm{ml}(7.69 \times$ $\left.10^{-7} M\right)$, respectively. Proximal tubular lumens were punctured with 5-7 $\mu \mathrm{m}$-tip diameter glass pipettes and $50 \mathrm{nl}$ of sample solution was infused in $1 \mathrm{~min}$.

\section{Data Acquisition and Processing}

All data acquisition was conducted with the Leica TCS-SL (software version, 2.871-6A, Leica Microsystems, Heidelberg, Germany). A Leica HCX PL APO $40 \times / 1.25-0.75$ oil immersion or a Leica PL APO $63 \times / 1.4$ oil immersion objective lens were used with free working distances of 100 and $60 \mu \mathrm{m}$, respectively. An argon laser was used to excite Alexa Fluor 488-labeled proteins at $488 \mathrm{~nm}$. Prior to recording, the diameter of the pinhole, laser power, gain and offset of PMT were set optimally to decrease the background signals, caused mainly by autofluorescence, to nearly zero. In order to reduce photobleaching and photodamage, the image averaging method was not used.

Immediately following micropuncture, the objective lens was changed to either the $40 \times$ or the $63 \times$ lens, focus was set through the eyepiece of the microscope using the autofluorescence of proximal tubules and scanning was started in reflection mode and/or fluorescence mode. A cover glass was placed between the surface of the kidney and the objective lens to obtain a stable image. In single scanning experiments, the areas of interest were scanned at the speed of $400 \mathrm{~Hz}$ and recorded by $512 \times 512$ pixels. In time-series experiments, scanning was conducted in fluorescence mode at the speed of $1,000 \mathrm{~Hz}$ and recorded by $256 \times 256$ pixels. The interval between each scanning was one second.

All single images were saved in TIFF format. Fluorescence and reflection images were merged in Adobe Photoshop (version 6.0). Multiple TIFF files in time-series experiment were converted to individual bitmap files and movies were produced using Microsoft VidEdit (version 1.1).

\section{Results}

\section{Basic Assessment of the in vivo Confocal Microscope System}

A representative image of proximal tubules in reflection mode is shown in figure 2. Proximal tubules were identified by an intensive reflection signal from the microvillus brush border. The height of the proximal tubule single layer of cuboidal cells was approximately $10-15 \mu \mathrm{m}$ and the lumen diameter was approximately $20-30 \mu \mathrm{m}$.

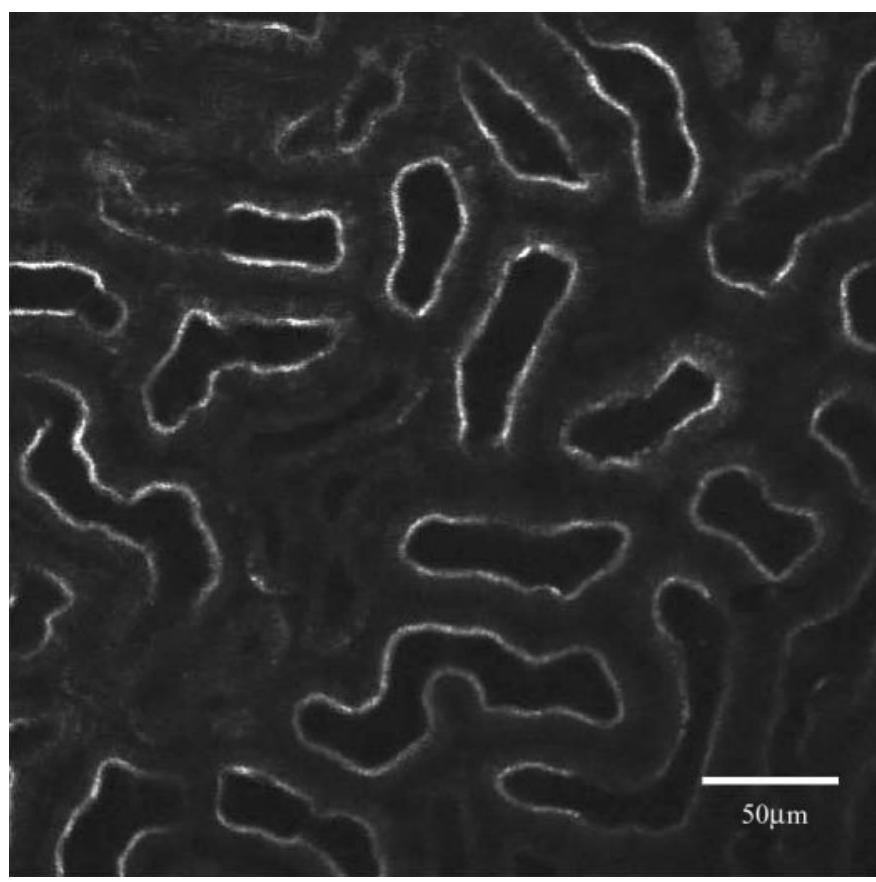

Fig. 2. Superficial proximal tubules in reflection mode. Superficial proximal tubules of the kidney were scanned with the $\times 40$ objective at the speed of $400 \mathrm{~Hz}$. The diameter of pinhole was set to 1.00 in airy unit. The intensive reflection of the brush border and open lumen can be seen.

To evaluate the contribution of a cover glass for the stability of the scanning field, time-series recordings every two seconds were conducted in reflection mode with and without cover glass. Focus was set around the center of superficial proximal tubular lumens in the horizontal plane using the $\times 40$ objective lens. With cover glass, movement of the brush border in the z-direction was restrained and acceptable while small movements in the xy-plane were observed. However, without cover glass, large movements of brush border in xyz-directions were observed and the brush border sometimes moved out of the focal plane (fig. 3). The advantage of the cover glass was dependent on the depth of the focal plane and greater in deeper planes. Two movies showing microscope recordings of the same proximal tubule with and without cover glass can be found at http://www.confocal.au.dk/.

Alexa Fluor 488-labeled RSA was microinfused into proximal tubules of interest and the area was scanned 30 min after microinfusion. Using the $40 \times$ objective lens, basic microscope settings were: PMT-gain 60\%; PMT offset 0 ; pinhole 1.98 , resulting in a $4.6 \mu \mathrm{m}$ theoretical optical section thickness. A micrograph obtained with this set- 
Fig. 3. Contribution of cover glass on stability of focal plane. Images scanned in reflection mode were converted inversely and four representative images under each condition were combined. Focus was kept in a pre-set plane with the cover glass and condensed signals of brush border means that the area of scanning was stable (A). On the contrary, signals of the brush border disappeared in some parts and were seen double in other parts for images obtained without cover glass (B).
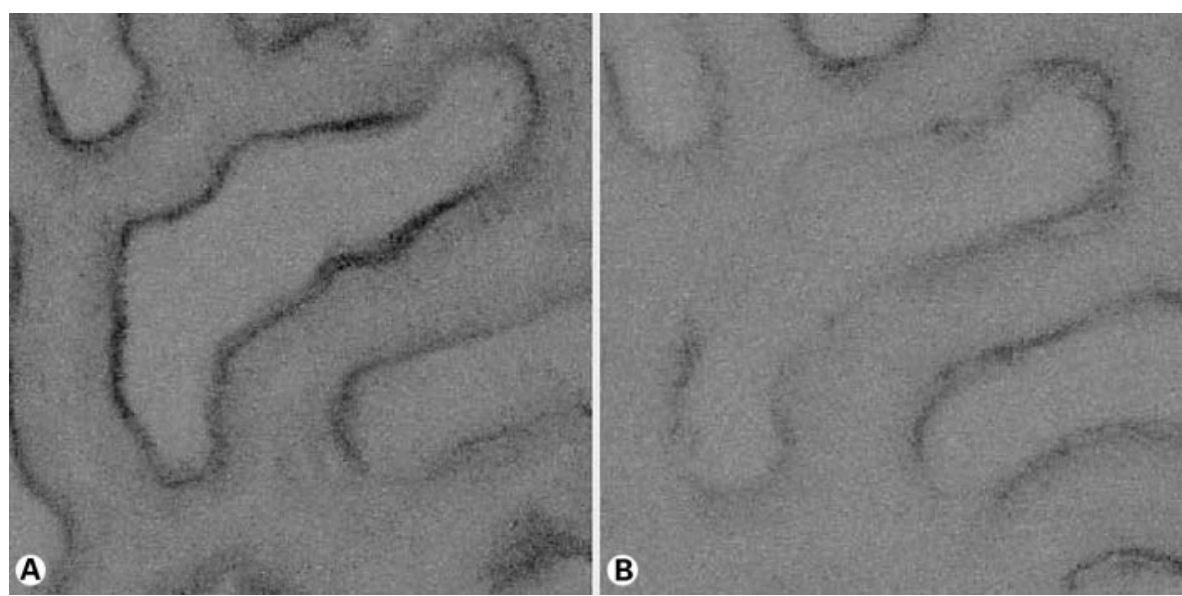

Fig. 4. Image in fluorescence mode under basic scanning condition. A proximal tubule was microinfused with Alexa Fluor 488labeled RSA. The area microinfused was scanned 30 min after finishing micropuncture. The brush border of the proximal tubules is seen in A. Green signals of Alexa Fluor 488-labeled RSA are seen only in proximal tubules micropunctured as a punctuate label (B). Objective lens, $\times 40$.
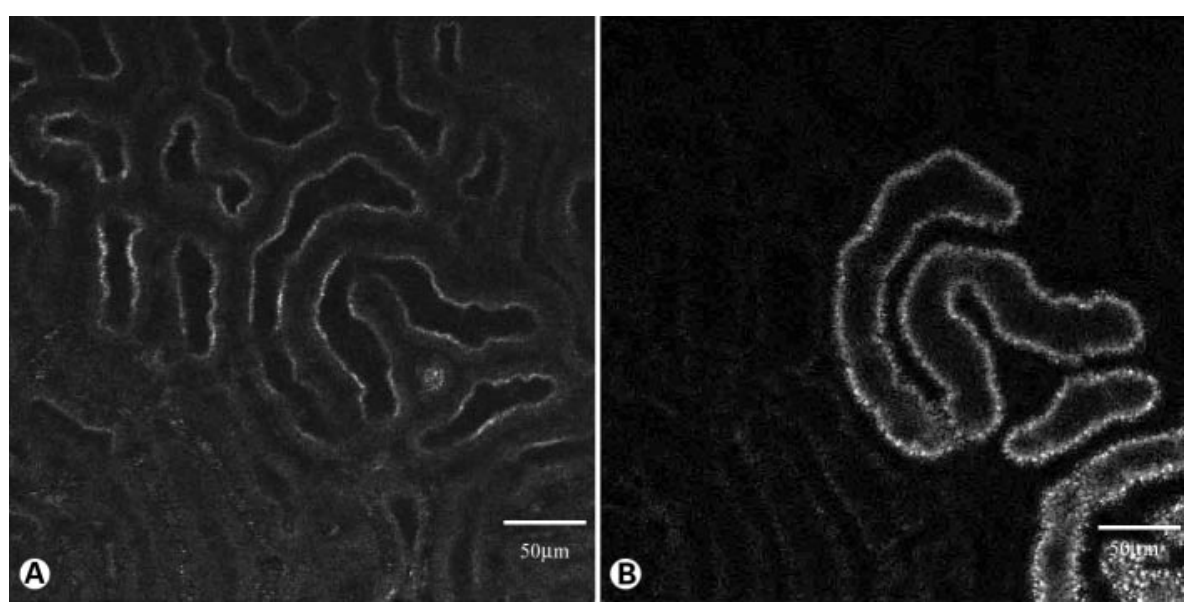

ting is shown in figure 4 . The focal plane was set by reflection mode (fig. 4A) and the same plane was scanned sequentially in fluorescent mode. Under these conditions, the signals of Alexa Fluor 488 were detected while autofluorescent signals were hardly observed (fig. 4B). Using the $63 \times$ objective lens, basic settings were: PT-gain $60 \%$; PMT-offset 0 ; pinhole 2.88 , resulting in a $7.5 \mu \mathrm{m}$ theoretical optical section thickness.

To determine maximal scanning depth within the kidney, signals from microinjected Alexa Fluor 488-labeled RSA were collected at the focal planes $0,25,50,75$ and $100 \mu \mathrm{m}$ from the surface of the kidney. The distance between the kidney surface and the focal plane was measured by using the display for position of the microscope stand. Figure 5 shows signals at 0,25 and $50 \mu \mathrm{m}$ below the surface. Intensively labeled endocytic vesicles were observed $25 \mu \mathrm{m}$ below the surface. At $50 \mu \mathrm{m}$ below the surface the signals in general were weaker, but strong enough to be detected. Only diffuse weak signals were detected at the plane $100 \mu \mathrm{m}$ below the surface and detection limit with maximum laser power was around $130 \mu \mathrm{m}$ (data not shown).

\section{Internalization of Alexa Fluor 488-Labeled Protein}

Figure 6 shows time-course images of internalization of microinjected Alexa Fluor 488-labeled RSA in proximal tubular cells. The area of interest was scanned sequentially in reflection and fluorescence mode and merged. Four minutes after finishing micropuncture, labeled RSA was observed on the brush border of proximal tubules (fig. 6A). Following another $4 \mathrm{~min}$, signals of Alexa Fluor 488-labeled RSA accumulated within the tubules in a punctuate fashion, presumably representing internalization into endosomes close to the brush border of proximal tubules (fig. 6B). After an additional $2 \mathrm{~min}$, these vesicles had moved to a deeper part of the tubular 


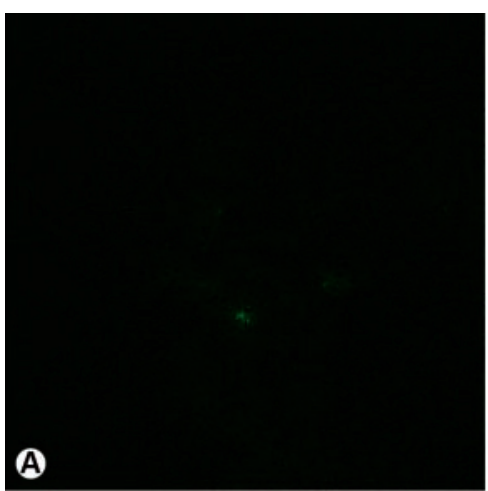

$0 \mu \mathrm{m}$

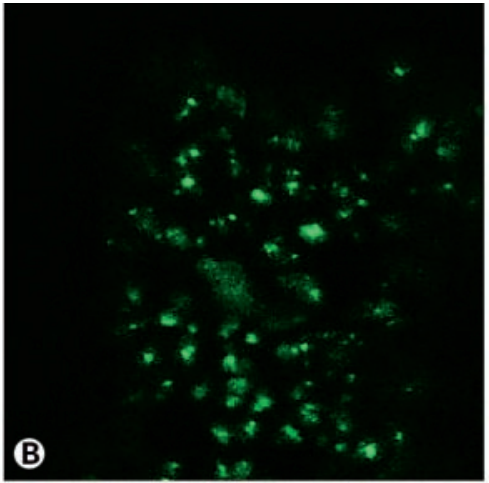

$25 \mu \mathrm{m}$

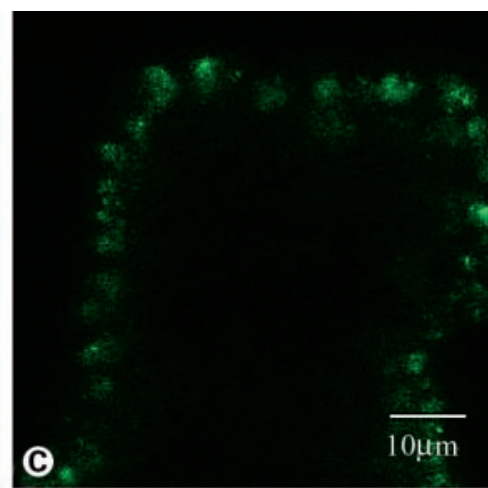

$50 \mu \mathrm{m}$

Fig. 5. Difference of signal intensity between planes at various depths. The proximal tubule microinfused with Alexa Fluor 488-labeled RSA was scanned 30 min after micropuncture. Signals were collected at planes $0(\mathbf{A}), 25,50 \mu \mathrm{m}$ from the surface of the kidney. High intensity signals are recognized at $25 \mu \mathrm{m}$ (B). Weaker but still reasonable intensity of signals was obtained from at $50 \mu \mathrm{m}(\mathbf{C})$.

Fig. 6. Time-course of internalization of $\mathrm{Al}-$ exa Fluor 488-labelled RSA in proximal tubules. The area was scanned in reflection mode and fluorescence mode sequentially. Fluorescent images were merged with corresponding reflection images. A Four minutes after micropuncture, signals of Alexa Fluor 488-labelled RSA are observed at the brush border in proximal tubules. No punctuate accumulation of albumin is seen. B Eight minutes after micropuncture, RSA had accumulated in endosomes visualized as punctuate accumulations of Alexa Fluor 488 which appeared below the brush border. C Ten minutes after micropuncture, punctuate accumulations presumably originating from endosomes had moved to a deeper zone in proximal tubules. D Fifteen minutes after micropuncture, punctuate accumulations are seen in a far more deeper zone, probably at least in part representing accumulation in lysosomes. Objective lens, $\times 63$.
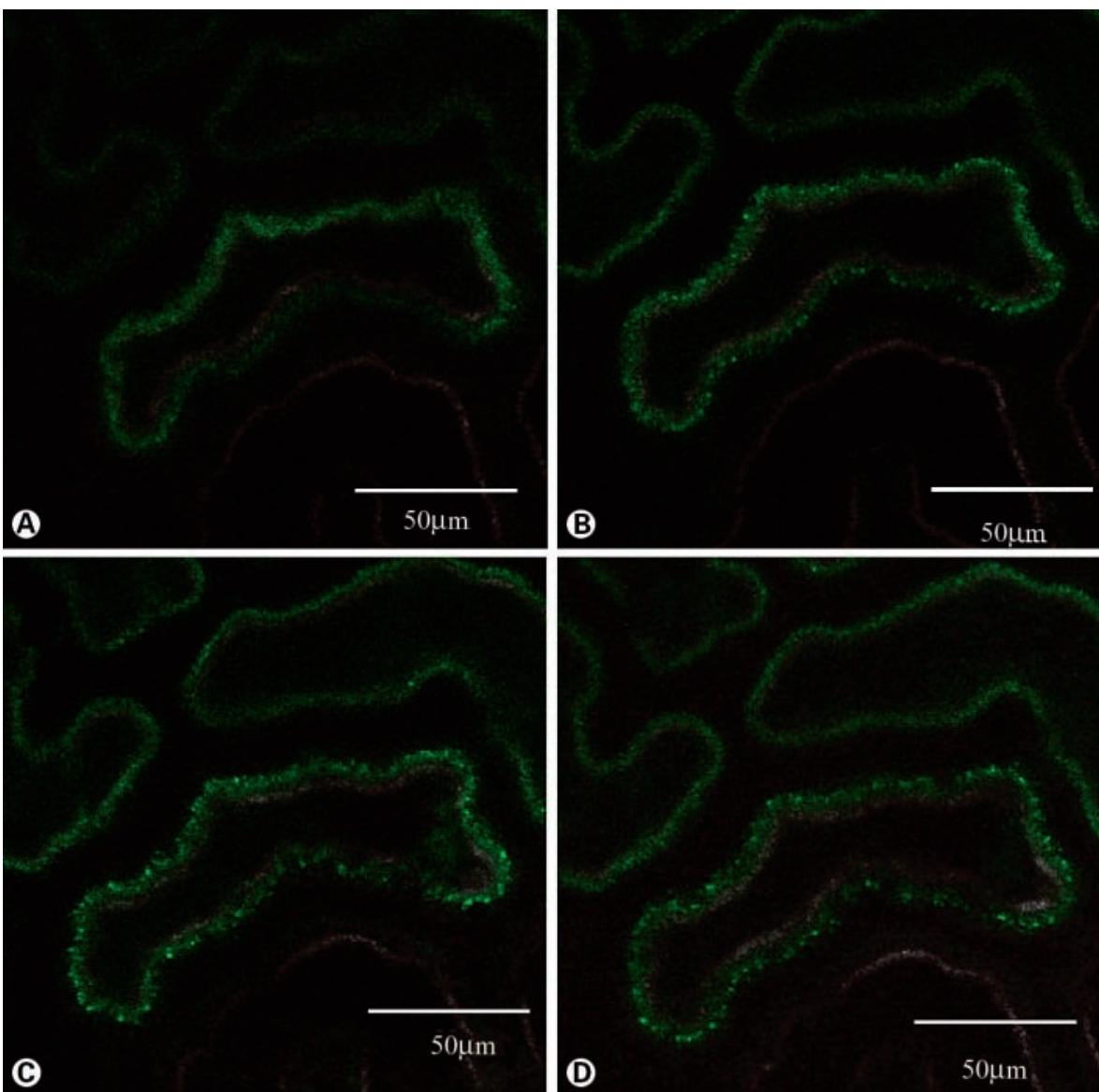

(D) 
Fig. 7. Micrograph of internalized RSA in fixed tubule. The proximal tubule was microinfused with Alexa Fluor 488-labeled RSA. The tubule microinfused was fixed with $2 \%$ paraformaldehyde $30 \mathrm{~min}$ after micropuncture. The whole kidney was excised and further fixed with $2 \%$ paraformaldehyde overnight. The micrograph shows punctuate signals of RSA in proximal tubule (A). Magnified image of the area flamed by white square is shown in B. $\mathrm{N}$ indicates nucleus and punctuate accumulations seen around the nucleus probably represent lysosomal accumulation.

Fig. 8. Internalization of albumin and transferrin in proximal tubules. The images were recorded $30 \mathrm{~min}$ after micropuncture. A, B Internalization of Alexa Fluor 488-labeled RSA. C, D Internalization of Alexa Fluor 488-labeled Tf. Similar punctuate accumulations are found in all images. Objective lens, $\times 63$.
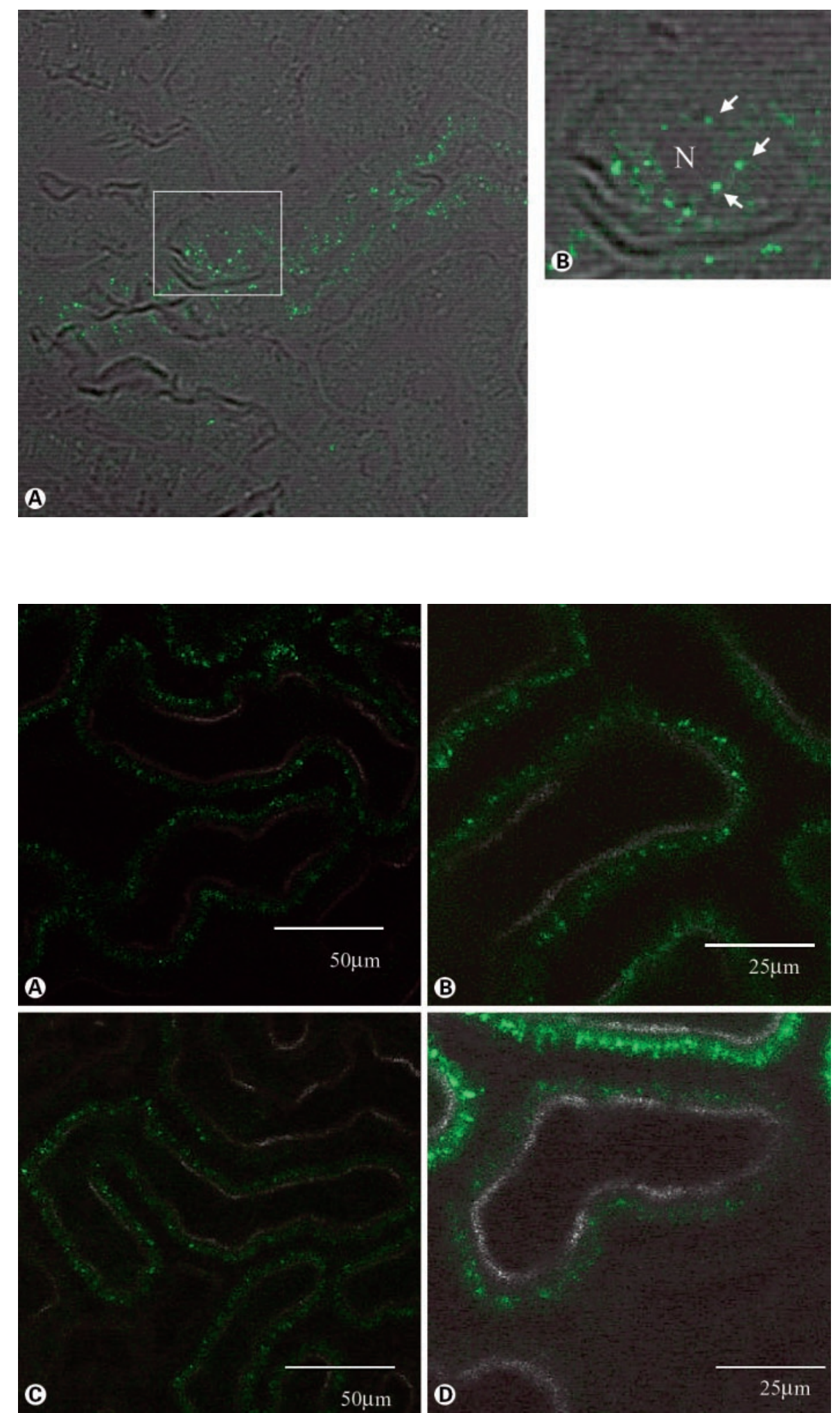

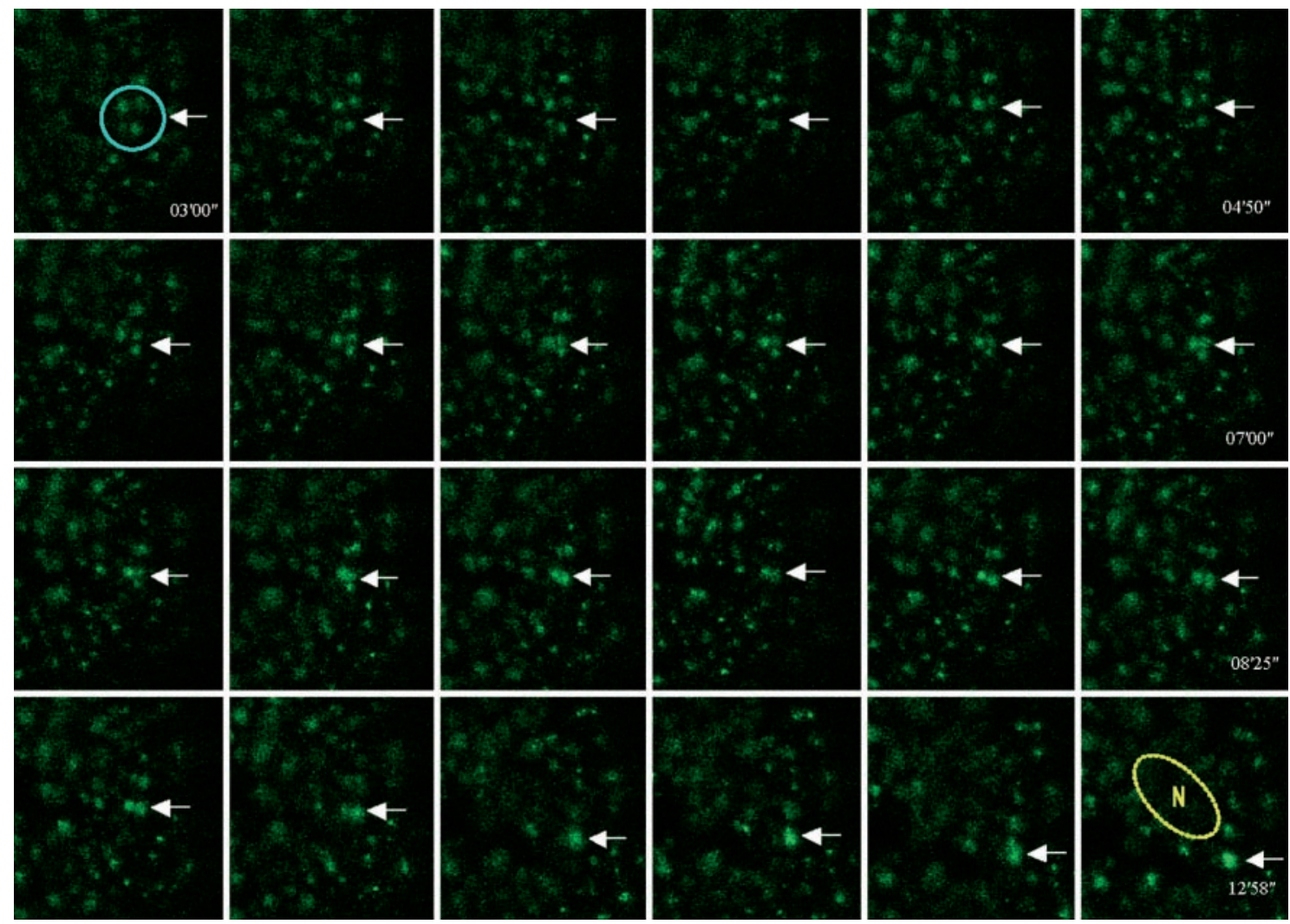

Fig. 9. Time-series images of albumin-internalization in proximal tubule. The proximal tubule was microinfused with Alexa Fluor 488-labeled RSA. Recording was started 3 min after micropuncture. The area was scanned at a speed of $1,000 \mathrm{~Hz}$ and images were recorded by $256 \times 256$ pixels. The pinhole was opened to 4.87 airy unit. With time the three vesicles in light blue circle fuse into one. Yellow circle indicates nucleus. Objective, $\times 40$.

cells (fig. 6C). At 15 min, steady accumulations were seen in an even deeper zone, probably representing lysosomes (fig. 6D). Figure 7 illustrates the internalization of RSA from a tubule fixed $30 \mathrm{~min}$ after microinfusion. Accumulation of Alexa Fluor 488-labeled RSA was concentrated close to the nucleus, presumably in lysosomes. Alexa Fluor 488-labeled Tf was internalized similarly to labeled RSA (fig. 8). The fluorescence signal of Tf was stronger compared to that of RSA. The greater labeling efficiency of $\mathrm{Tf}$ as well as higher binding affinity to the endocytic receptor cubilin [17] may be responsible for this difference in signal intensity.

To examine vesicular transport in proximal tubules using the in vivo microscope system, the focus was set at cells located in the superficial wall of superficial proximal tubules. This plane was about $25 \mu \mathrm{m}$ below the surface (see also fig. 5). Due to small movements of the kidney, it was necessary to adjust focus manually during recording. Representative images of vesicular fusion observed in time-series recordings and selected from multiple TIFF files are shown in figure 9. The recording was started 3 min after finishing microinfusion of labeled RSA. Three vesicular accumulations of label were recognized and marked by a light blue circle. These vesicles fused into two and finally into one structure within $13 \mathrm{~min}$. The nucleus of the proximal tubular cell was located in the area indicated by the yellow circle. A supplementary movie showing internalization of Alexa Fluor 488-labeled RSA can be found at http://www.confocal.au.dk/.

\section{Discussion}

The present study shows that in vivo confocal microscopy allows visualization of endocytosed Alexa Fluor 488labeled RSA and $\mathrm{Tf}$ in proximal tubules of intact rat kidney. In addition, we have demonstrated vesicular fusion. While fusion and fission of vesicles within the endocytic 
and recycling pathway has been intensively studied in vitro and in cell culture, the dynamics of the endocytic apparatus have not been fully visualized in vivo. Proximal tubular cells reabsorb filtered proteins very efficiently within minutes after intravenous injection followed by lysosomal accumulation and degradation [17-20]. The rapid RSA accumulation shown in our time series experiments (fig. 9) is consistent with these results. Thus, CLSM is suitable for studies of intracellular transport of proteins and a potential tool for the analysis of proximal tubular cell function at the subcellular level in vivo.

Conventional wide-field fluorescent microscopy is not capable of high-resolution imaging of intracellular dynamics as microheterogeneity of thick samples causes scattering, absorption of light, and reduces light intensity. As whole specimens are illuminated, light from out-offocus planes degrades resolution. Three kinds of technology may eliminate unwanted light from out-of-focus planes: image deconvolution, CLSM, and TPLSM. For deconvolution, images are processed by computer algorithm requiring images with high signal-to-noise ratios [21]. This is unsuitable for the dynamic observation of living cells or intact organs since it is difficult to process recording and deconvolution simultaneously in addition to stabilizing the focal plane. In contrast, both CLSM and TPLSM are prospective candidates for intravital microscopy of intact organs. In CLSM the whole thickness of the specimen is illuminated and the detector pinhole eliminates lights from out-of-focus planes. In TPLSM light from out-of-focus planes is not generated as excitation is confined to the vicinity of the focal point.

Observations by CLSM are limited by the illumination depth of the laser. When thick samples are illuminated with infrared laser light, CLSM is only able to collect light within 100-200 $\mu \mathrm{m}$ and resolution is decreased [22]. The maximum distance from the surface of the kidney, from which we could obtain a fluorescent signal with maximum laser power, was $130 \mu \mathrm{m}$. However, since the free working distance of the $40 \times$ objective lens is at maximum $100 \mu \mathrm{m}$, observations below this results in substantial pressure from the lens onto the kidney surface. Thus, only superficial proximal tubules can be studied in normal kidney. The near-infrared laser used for multophoton microscopy penetrates deeper into scattering specimens than commonly used visible excitation [23]. TPLSM increases imaging penetration depth at least two-fold compared to CLSM [24].

Recently, Dunn et al. [14] reported the use of TPLSM for studying intact rat kidney proximal tubule function. After administration of fluorescent probes by intravenous injection or micropuncture technique, they obtained images with excellent resolution, including images of glomerular filtration of fluorescence labeled dextran and gentamicin, and time-course images showing internalization of these compounds in proximal tubules. Limitations in TPLSM includes relatively slow scanning speed which may impede real time observation of intracellular dynamics and less prevalence of the necessary equipment making CLSM an attractive alternative. Several methodological problems, however, need to be addressed. Time-series images of Alexa Fluor 488-labeled RSA internalization, showed vesicular fusion, but the stability of the scanning field in these experiments was not sufficient to follow single vesicular movements. This instability, caused by microcirculation, could in part be overcome by the use of a cover glass on top of the convex kidney surface. Theoretically, the objective lens never touches the cover glass at a focal plane as deep as $100 \mu \mathrm{m}$ below the surface of the kidney. However, since the kidney surface is convex and the cover glass is plane, an increasing free distance between the kidney surface and the cover glass appears in the periphery. Thus, when focusing away from the center of the cover glass, the working distance above the cover glass is reduced and eventually the objective lens touches the cover glass applying a mild pressure. The stability of the scanning field by the cover glass may be due to this pressure. This explanation implies that an appropriate selection of proximal tubules can result in acceptable stability to the scanning field. In addition, vesicles may move in the z-dimension out of the focal plane. Thus adjacent planes need to be scanned sequentially requiring high scanning speed reducing the fluorescent intensity. The simplest alternative way to overcome this is to open the pinhole. Thus, in our time-series experiment to follow single vesicles, the pinhole was opened to 4.87 airy unit resulting in a $12.2-\mu \mathrm{m}$ optical section thickness nearly equal to the height of a single layer of proximal tubule cells, resulting in the loss of resolution in the z-dimension.

A potential problem of in vivo CLSM is laser phototoxicity which may be responsible for the limited number of vesicular fusion images seen in figure 9. TPLSM is less phototoxic but encounters the same problem. Cell culture experiments have established this effect and it was shown that the addition of ascorbic acid to the medium may reduce phototoxicity [25], but this method is impractical in intact organ experiments. Thus, it will be necessary to evaluate cellular damage (morphological and functional changes) after laser illumination, e.g. ultrastructural changes in intracellular organelles and reabsorption of proteins in illuminated proximal tubules. 
In conclusion, by optimizing the technique we were able to visualize the endocytosis of microinjected RSA and $\mathrm{Tf}$ in situ by CLSM. Both ligands were initially observed at the brush border, then internalized by proximal tubule cells, and accumulated in lysosomes. Furthermore, we could identify the fusion of endocytic vesicles in time-lapse images. The observations suggest that in vivo confocal microscopy will contribute to further progress of renal physiology studies.

\section{Acknowledgements}

This work was supported by grants from the Danish Medical Research Council, the Novo Nordic Foundation, the Danish Biotechnology Program, the Velux Foundation and the University of Aarhus Research Foundation. The study was presented in part at the 36th Annual Meeting of the American Society of Nephrology, San Diego, California, 2003. The authors would like to thank Inger Blenker Kristoffersen, Pia Kamuk Nielsen and Hanne Sidelmann for excellent technical assistance.

\section{References}

1 Rizzuto R, Brini M, Pizzo P, Murgia M, Pozzan T: Chimeric green fluorescent protein as a tool for visualizing subcellular organelles in living cells. Curr Biol 1995;5:635-642.

$>2$ Baumann CT, Lim CS, Hager GL: Simultaneous visualization of the yellow and green forms of the green fluorescent protein in living cells. J Histochm Cytochem 1998;46:10731076.

$\checkmark 3$ Messerli JM, Perriard JC: Three-dimensional analysis and visualization of myofibrillogenesis in adult cardiomyocytes by confocal microscopy. Microsc Res Tech 1995;30:521-530.

$\checkmark 4$ Sönnichsen B, Renzis SD, Nielsen E, Rietdorf $\mathrm{J}$, Zerial M: Distinct membrane domains on endosomes in the recycling pathway visualized by multicolour imaging of Rab4, Rab5, and Rab 11. J Cell Biol 2000;149:902-913.

$\checkmark 5$ Roberts RL, Barbieri MA, Pryse KM, Chua M, Morisaki JH, Stahl PD: Endosome fusion in living cells overexpressing GFP-rab5. J Cell Sci 1999; 112:3667-3675.

6 Huang F, Nesterov A, Carter RE, Sorkin A: Trafficking of yellow-fluorescent-protein-tagged $\mu 1$ subunit of clathrin adaptor AP-1 complex in living cells. Traffic 2001;2:345-357.

7 Sako Y, Minoguchi S, Yanagida T: Single-molecule imaging of EGFR signalling on the surface of living cells. Nat Cell Biol 2000;2:168172.

$\checkmark 8$ Kleinfeld D, Mitra PP, Helmchen F, Denk W: Fluctuations and stimulus-induced changes in blood flow observed in individual capillaries in layers 2 through 4 of rat neocortex. Proc Natl Acad Sci USA 1998;95:15741-15746.
Grayson MH, Chaplin DD, Karl IE, Hotchkiss RS: Confocal fluorescent intravital microscopy of the murine spleen. J Immunol Methods 2001;256:55-63.

10 Huzaira M, Rius F, Rajadhyaksha M, Anderson RR, Gonzalez S: Topographic variations in normal skin, as viewed by in vivo reflectance confocal microscopy. J Invest Dermatol 2001; 116:846-852.

$>11$ Jester JV, Lee YG, Li J, Chakravarti S, Paul J, Petroll WM, Cavanagh HD: Measurement of corneal sublayer thickness and transparency in transgenic mice with altered corneal clarity using in vivo confocal microscopy. Vision Res 2001;41:1283-1290.

12 Schneider P, Foitzik T, Kahrau S, Podufal A, Buhr H-J: An experimental rat model for studying pulmonary microcirculation by in vivo videomicroscopy. Microvasc Res 2001; 62:421-434.

13 Boyde A, Capasso G, Unwin RJ: Conventional and confocal epi-reflection and fluorescence microscopy of the rat kidney in vivo. Exp Nephrol 1998;6:398-408.

14 Dunn KW, Sandoval RM, Kelly KJ, Dagher PC, Tanner GA, Atkinson SJ, Bacallao RL, Molitoris BA: Functional studies of the kidney of living animals using multicolour two-photon microscopy. Am J Physiol Cell Physiol 2002; 283:C905-C916.

15 Christensen EI, Rennke HG, Carone FA: Renal tubular uptake of protein: Effect of molecular charge. Am J Physiol 1983;244:F436-F441.

16 Birn H, Nielsen S, Christensen EI: Internalization and apical-to-basolateral transport of folate in rat kidney proximal tubule. Am J Physiol 1997;272:F70-F78.
7 Christensen EI, Birn H: Megalin and cubilin: Multifunctional endocytic receptors. Nat Rev Mol Cell Biol 2002;3:256-266.

18 Christensen EI: Rapid protein uptake and digestion in proximal tubule lysosomes. Kidney Int 1976;10:301-310.

19 Maunsbach AB: Absorption of ${ }^{125}$ I-labeled homologous albumin by rat kidney proximal tubule cells: A study of microperfused single proximal tubules by electron microscopic autoradiography and histochemistry. J Ultrastruct Res 1966;15:197-241.

20 Maunsbach AB, Christensen EI: Functional ultrastructure of the proximal tubule; in Windhager EE (ed): Handbook of Physiology. Renal Physiology, section 8 (Renal Physiology, vol I). New York, Oxford University Press, 1992, pp 41-107.

21 White JG, Squirrell JM, Eliceiri KW: Applying multiphoton imaging to the study of membrane dynamics in living cells. Traffic 2001;2:775780 .

22 Tadrous PJ: Methods for imaging the structure and function of living tissues and cells. 3. Confocal microscopy and micro-radiology. J Pathol 2000;191:345-354.

23 So PT, Dong CY, Masters BR, Berland KM: Two-photon excitation fluorescence microscopy. Annu Rev Biomed Eng 2000;2:399-429.

24 Centonze VE, White JG: Multiphoton excitation provides optical sections from deeper within scattering specimens than confocal imaging. Biophys J 1998;75:2015-2024.

25 Terasaki M, Dailey ME: Confocal microscopy of living cells; in Pawley JB (ed): Handbook of Biological Confocal Microscopy, ed 2. New York, Plenum, 1995, pp 327-346. 